\title{
The Impact of Sociodemographic Factors on Knowledge of Cardiac Procedures
}

\author{
Samara Lipsky ${ }^{1}$, Michael Bohnen ${ }^{2}$, Janice Barnhart ${ }^{3}$ \\ ${ }^{1}$ Department of Psychology, Yeshiva University, Bronx, USA; ${ }^{2}$ Columbia University, New York, USA; ${ }^{3}$ Department of Epidemi- \\ ology and Population Health, Albert Einstein College of Medicine, Bronx, USA. \\ Email: SamaraLipsky@alum.emory.edu
}

Received July $19^{\text {th }}, 2010$; revised July $26^{\text {th }}, 2010$; accepted August $3^{\text {rd }}, 2010$.

\begin{abstract}
Background: This paper investigates the extent to which sociodemographic factors are associated with knowledge of cardiac procedures in a sample of study participants treated for coronary heart disease (CHD). Research indicates the importance of knowledge of CHD and its associated risks in order to prevent CHD. However, quantification of knowledge levels among individuals undergoing cardiac procedures to treat CHD has not been well documented. Method: Using a cross-sectional design, 156 participants, diverse in race/ethnicity, age, and sex, underwent elective cardiac catheterization for the evaluation of chest pain and/or angina. Participants completed surveys regarding medical history, sociodemographic information, and knowledge of cardiac procedures. Ninety-five of these individuals, with clinically significant $C H D$, were recommended by their physician to undergo a coronary revascularization procedure [percutaneous transluminal coronary angioplasty (PTCA) or coronary artery bypass graft (CABG)]. These individuals completed additional knowledge assessment surveys. Results: The overall knowledge scores for those undergoing coronary angiography were suboptimal $(M$ score $=4.6$ out of 8$)$. Older aged $(>65)$, male, married, white, college-educated participants demonstrated greater knowledge of cardiac catheterization procedures (all $p$ values $<0.05$ ). Knowledge scores were greater among those revascularized than among participants undergoing coronary angiography. Conclusions: Health professionals should provide general information about CHD treatment and interventions, especially among women and ethnic minorities.
\end{abstract}

Keywords: Coronary Heart Disease, Knowledge, Cardiac Procedure

\section{Introduction}

Coronary heart disease (CHD) is the primary cause of death for adults in the United States [1,2]. Numerous reports outline disparities in knowledge of cardiovascular disease among different sociodemographic groups for individuals with CHD based on race/ethnicity and sex $[3,4]$. For example, Woodard and colleagues (2005) found that African Americans demonstrated less knowledge regarding risk factors for cardiovascular disease, compared to Caucasians. The lesser knowledge that African Americans demonstrated regarding risk factors of cardiovascular disease may directly correlate with the finding that African American women with multiple cardiac risk factors underestimated their risk for heart disease [5]. Furthermore, Mosca and colleagues (2000) surveyed over 1,000 women and found that only $8 \%$ recognized CHD or stroke as their greatest health concern and less than one-third identified CHD as a leading cause of death. Thus, lack of knowledge regarding personal risk of CHD may impact decision-making regarding risk prevention, and this impact may be heightened among sociodemographic groups under-educated on the risks and prevention of cardiovascular disease.

Few studies have examined the origin of the disparities in knowledge of CHD based on race/ ethnicity and sex among individuals with CHD $[3,4]$. The root cause might include the effect of a previously unmeasured non-clinical factor: knowledge of the risks and benefits of cardiac procedures, including coronary angiography and revascularization (i.e. percutaneous transluminal coronary angioplasty [PTCA] or coronary artery bypass graft [CABG]) [3]. Determining if knowledge affects individuals' decision-making might aid in understanding disparities in the provisions of the use of coronary angiography or revascularization [6,7]. Moreover, if knowledge levels of these cardiac procedures vary along sociodemographic lines, the root of the disparity in know- 
ledge of individuals with cardiovascular disease may be targeted further.

\section{Method}

\subsection{Participants}

Individuals who met the following criteria were invited to participate in the study: 1) $\geq 40$ years of age; 2) undergoing angiography for chest pain/pressure and/or angina equivalents; and 3) able to give informed consent. Out of 217 individuals eligible for the study, 181 agreed to participate, and we obtained complete data from 156 participants. These 156 participants had their knowledge level of the catheterization they were about to undergo assessed. Ninety-five of these individuals had their knowledge level of their subsequent revascularization procedure assessed via bedside survey.

\subsection{Procedure}

Knowledge of cardiac procedures was investigated among male and female participants scheduled for coronary angiography at an academic medical center in the Bronx. These individuals were identified weekly using the logs at this medical center. Trained interviewers abstracted information from medical log books to locate eligible participants. Once located, individuals were asked to be interviewed at bedside and permission was granted to access their health information.

\subsection{Measures}

Following the baseline interview, medical record abstractors used standardized forms to collect detailed information on medical history, angiography results, and angina severity as classified by the Canadian Cardiovascular Society [8]. These forms consisted of the following items: age, race/ethnicity, sex, marital and employment status, level of education, insurance, medical history, angiography results, and treatment received. In addition, all respondents completed eight true-false questions regarding knowledge of coronary angiography. One point was assigned to each correct response (possible score 0 to 8). All individuals with clinically significant CHD that underwent a revascularization procedure (i.e. PTCA or CABG) also completed a survey pertaining to their respective procedures. These surveys contained a mix of true/false and multiple choice questions (possible scores for $\mathrm{CABG}=0$ to 10 ; for $\mathrm{PTCA}=0$ to11). A higher mean score indicated that the individual had greater knowledge of his or her corresponding procedure.

\subsection{Statistical Analysis}

We used descriptive statistics (e.g. percentages, means, standard deviations) to characterize the study population.
Univariate analyses allowed us to calculate the crude rates for coronary angiography and revascularization (i.e. PTCA and CABG) according to the patients' demographic characteristics. We determined significant associations with chi-square tests for categorical variables (e.g. sex) and student's t-tests for continuous variables (e.g. age, knowledge scores). Marital status was coded as married and not married (single, separated, divorced and widowed). Age was dichotomized to $\leq 65$ years and $>65$ years based on the study population. Race/ethnicity was coded as white and non-white (African American, Hispanic, Native American, Asian, and other). Level of education was coded as college degree (college, graduate school) and no college degree (high school, high school diploma, some college experience but no degree obtained). We calculated knowledge of cardiac procedures by summing the number of correct responses to the angiography, PTCA, and CABG questions.

\section{Results}

\subsection{Sociodemographic Characteristics}

The study's sample included 156 participants ranging in age from 42 to 96 (mean $=69.2 ; \mathrm{SD}=9.5$ years) and the majority were women (93 females, $59.6 \%$ of participants). The major racial/ethnic groups were white (56\%), non-Hispanic black (18\%), and Hispanic (16\%). The majority of participants had private insurance $(60 \%)$ or Medicare (20\%). Most individuals had at least two risk factors for CHD, hypertension and hypercholesterolemia the most prevalent among them. Chronic stable angina (moderate-severe) was the most common presenting symptom. Fifty-eight percent of the participants had mild-moderate CHD (i.e. at least 50\% blockage of a main heart artery) and PTCA was the more common revascularization procedure performed (54\%). Demographic characteristics and clinical data of the study population are displayed in Table $\mathbf{1 .}$

\subsection{Knowledge of Angiography}

Knowledge levels of coronary angiography varied significantly according to an individual's sociodemographic background. The overall mean score for the eight angiography knowledge questions was $4.6(\mathrm{SD}=1.7)$ as shown in Table 2. Scores ranged from $0(n=2)$ to $8(n=$ 3). Participants who were over 65 years of age, male, married, college educated, and white had higher mean knowledge scores for cardiac catheterizations than their respective counterparts ( $\mathrm{p}<0.05$ for all comparisons). Participants who were employed were more knowledgeable about their procedures as well. 
Table 1. Characteristics of Study Population (Total, n=154).

\begin{tabular}{|c|c|c|}
\hline Variable & $\operatorname{Mean}( \pm \mathrm{SD})$ & Percentage \\
\hline Age & $69.2(9.5)$ & \\
\hline \multicolumn{2}{|l|}{ Female } & 59.6 \\
\hline \multicolumn{2}{|l|}{ Married } & 62.2 \\
\hline \multicolumn{2}{|l|}{ Employed } & 42.3 \\
\hline \multicolumn{3}{|c|}{ Race/Ethnicity } \\
\hline \multicolumn{2}{|l|}{ Caucasian } & 56.4 \\
\hline \multicolumn{2}{|c|}{ Non-Hispanic Black } & 17.9 \\
\hline \multicolumn{2}{|l|}{ Hispanic } & 16.0 \\
\hline \multicolumn{2}{|l|}{ Other ${ }^{1}$} & 9.0 \\
\hline \multicolumn{3}{|l|}{ Education } \\
\hline \multicolumn{2}{|l|}{ College } & 67.3 \\
\hline \multicolumn{2}{|l|}{ No college } & 31.4 \\
\hline \multicolumn{3}{|l|}{ Insurance } \\
\hline \multicolumn{2}{|l|}{ Private } & 60.2 \\
\hline \multicolumn{2}{|l|}{ Medicare } & 19.9 \\
\hline \multicolumn{2}{|c|}{ Medicaid or Self-pay } & 17.1 \\
\hline \multicolumn{2}{|l|}{ Other ${ }^{2}$} & 1.8 \\
\hline \multicolumn{3}{|c|}{ Coronary Risk Factors } \\
\hline \multicolumn{2}{|l|}{ Diabetes } & 48.8 \\
\hline \multicolumn{2}{|c|}{ Hypertension } & 76.8 \\
\hline \multicolumn{2}{|c|}{ Hypercholesterolemia } & 74.0 \\
\hline \multicolumn{2}{|l|}{ Smoker } & 29.3 \\
\hline \multicolumn{2}{|c|}{ Two or more } & 79.0 \\
\hline \multicolumn{3}{|c|}{ Clinical Diagnosis } \\
\hline \multicolumn{2}{|c|}{ Atypical chest pain } & 8.8 \\
\hline \multicolumn{2}{|c|}{ Chronic stable angina Class I/II } & 17.1 \\
\hline \multicolumn{2}{|c|}{ Chronic stable angina Class III/IV } & 43.1 \\
\hline \multicolumn{2}{|c|}{ Unstable angina } & 9.9 \\
\hline \multicolumn{2}{|c|}{ Recent myocardial infarction } & 21.0 \\
\hline \multicolumn{3}{|c|}{ Angiography Results } \\
\hline Mild to $\mathrm{mc}$ & ( 1 or 2 vessels) & 57.5 \\
\hline Severe dis & s or left main) & 42.5 \\
\hline Treatment & & \\
\hline Medical & & 16.6 \\
\hline PTCA & & 54.1 \\
\hline $\mathrm{CABG}$ & & 28.7 \\
\hline
\end{tabular}

${ }^{1}$ Other includes: 7Asians, 6 of unknown ethnicity or race, and 1 Native American;

${ }^{2}$ Other includes: 1 government (VA) and 2 SSI.

\subsection{Knowledge of Revascularization Procedures}

Ninety-five individuals were revascularized $(\mathrm{CABG}=31$; PTCA =64). Participants that underwent a CABG were more knowledgeable regarding their procedure (mean score $=8.0$ out of $10, \mathrm{SD}=1.2$ ) than those undergoing PTCA $($ mean score $=7.9$ out of $11, \mathrm{SD}=1.7)$. There were
Table 2. Knowledge of coronary angiography by select patient characteristics ${ }^{1}$ (Total, $n=154$ ).

\begin{tabular}{ccc}
\hline Variable & Mean $( \pm$ SD) & Value $\mathrm{p}$ \\
\hline Age & & \\
$\leq 65$ years, $(\mathrm{n}=54)$ & $5.1(1.5)$ & \\
$>65$ years, $(\mathrm{n}=100)$ & $4.4(1.7)$ & $\mathrm{p}=0.018$ \\
Sex & & \\
Female, $(\mathrm{n}=60)$ & $3.9(1.9)$ & \\
Male, $(\mathrm{n}=89)$ & $5.1(1.4)$ & $\mathrm{p}<0.001$ \\
Married & & \\
Yes, $(\mathrm{n}=94)$ & $4.9(1.6)$ & \\
No, $(\mathrm{n}=56)$ & $4.2(1.7)$ & $\mathrm{p}=0.017$ \\
Race/Ethnicity & & \\
White, $(\mathrm{n}=84)$ & $4.9(1.7)$ & \\
Non-white, $(\mathrm{n}=65)$ & $4.2(1.5)$ & $\mathrm{p}=0.006$ \\
Employed & & \\
Yes, $(\mathrm{n}=64)$ & $4.9(1.5)$ & \\
No, $(\mathrm{n}=85)$ & $4.4(1.7)$ & $\mathrm{p}=0.052$ \\
Education & & \\
College, $(\mathrm{n}=46)$ & $5.0(1.6)$ & \\
No College, $(\mathrm{n}=102)$ & $4.4(1.6)$ & $\mathrm{p}=0.031$ \\
\hline
\end{tabular}

${ }^{1}$ Mean correct score (total of eight questions). Overall mean score $=4.6( \pm 1.7)$.

no significant demographic differences in knowledge scores among the participants that underwent CABG. However, married participants were more knowledge about PTCA than those who were single $(8.3[ \pm 1.5]$ vs. $7.3[ \pm 1.8] ; \mathrm{p}=0.026)$.

\section{Discussion}

General knowledge of coronary angiography was suboptimal, while revascularized individuals were more knowledgeable about their respective procedures (i.e. CABG or PTCA). There were no race/ethnicity or sex differences in knowledge or receipt of PTCA or CABG. Furthermore, we found significant differences in knowledge of coronary angiography according to age, sex, race/ethnicity, marriage and level of education.

Our finding that men and participants who identified themselves as white were overall more knowledgeable of their procedures may follow a cause-and-effect relationship, as men and whites undergo angiography more often than women and ethnic minorities, respectively [9]. The higher frequency by which males and white individuals undergo coronary angiography compared to females and ethnic minorities can in part be understood by noting that African Americans have been found to be less willing than white individuals to undergo these procedures [10]. This situation is not so clear-cut, though, as ethnic minrities may be offered coronary angiography less often, and thus may not undergo these procedures at the same 
high frequency. Nevertheless, a possible greater unwillingness of African Americans to undergo coronary angiography compared to white individuals corresponds with findings that African Americans are less likely than white individuals to feel that their physicians have a participatory style of decision making [11]. Whether African Americans demonstrate low knowledge of coronary angiography due to a lack of trust in their physician is an important consideration.

Most of our respondents were older individuals and had multiple cardiovascular risk factors. Therefore, they might have had a previous cardiac procedure. Moreover, most of our study participants were referred by their primary care physicians or cardiologists for their cardiac interventions. These two key characteristics of our study participants suggest that knowledge among participants may have varied depending on when in the course of their disease they interacted with a physician or subspecialist, or due to a systematic factor related to patient communication. Alternatively, the lower education level may have led to health illiteracy leading to lack of access to or integration of available knowledge. These variables warrant further study.

This study has limitations. We surveyed participants at one academic institution, thus our results may have limited generalizability. We did not detect significant differences in knowledge among the represented sociodemographic groups regarding CABG and PTCA. A larger sample size may have shed light on this uncertainty.

While we only found significant sociodemographic differences in levels of knowledge for coronary angiography and not for the revascularization procedures (CABG and PCTA) surveys, it is important to consider the nature of the questions we asked our study participants. While we tried to administer questions with a similar range of difficulty in all surveys, we cannot be fully confident that our questions offered a most precise gauge of knowledge for each procedure.

\section{Conclusions}

In this study, sociodemographic variables appeared to be associated with knowledge levels in individuals undergoing coronary angiography. These same variables did not produce significant differences in knowledge levels of individuals undergoing revascularization procedures. Earlier findings that knowledge of cardiovascular disease itself is low among under-educated sociodemographic groups may support our finding that these groups demonstrate low knowledge levels of related cardiac procedures. Disparity in knowledge among individuals undergoing coronary angiography warrants further study. Moreover, empowering individuals with CHD with greater knowledge of life-style or procedural treatments for CHD should enhance health promotion, which may improve individuals' quality of life before and after cardiac interventions. Adequate access to and integration of available knowledge becomes particularly important for individuals with CHD who may benefit from more timely access to medical care.

\section{REFERENCES}

[1] Center for Disease Control, "Heart Disease Facts: America's Heart Disease Burden,” 2006.

[2] L. Mosca, W. K. Jones, K. B. King, P. Ouyang, R. F. Redberg, M. N. Hill, et al., "Awareness, Perception, and Knowledge of Heart Disease Risk and Prevention among Women in the United States," Archives of Family Medicine, Vol. 9, No. 6, 2000, pp. 506-515.

[3] A. H. Christian, W. Rosamond, A. R. White and L. Mosca, "Nine-Year Trends and Racial and Ethnic Disparities in Women's Awareness of Heart Disease and Stroke: An American Heart Association National Study," Journal of Womens Health, Vol. 16, No. 1, 2007, pp. 68-81.

[4] H. M. Prendergast, E. B. Bunney, T. Roberson and T. Davis, "Knowledge of Heart Disease among Women in an Urban Emergency Setting," Journal of the National Medical Association, Vol. 96, No. 8, 2004, pp. 1027-1031.

[5] K. B. DeSalvo, J. Gregg, M. Kleinpeter, B. R. Pedersen, A. Stepter and J. Peabody, "Cardiac Risk Underestimation in Urban, Black Women," Journal of General Internal Medicine, Vol. 20, No. 12, 2005, pp. 1127-1131.

[6] J. M. Barnhart and S. Wassertheil-Smoller, "The Effect of Race/Ethnicity, Sex, and Social Circumstances on Coronary Revascularization Preferences: A Vignette Comparison," Cardiology in Review, Vol 14, No. 5, 2006, pp. 215-222.

[7] E. H. Bradley, S. A. McGraw, L. Curry, A. Buckser, K. L. King, S. V. Kasl, et al., "Expanding the Andersen Model: The Role of Psychosocial Factors in Long-Term Care Use," Health Services Research, Vol. 37, 2002, No. 5, pp. 1221-1242.

[8] L. Campeau, "Grading of Angina-Pectoris," Circulation, Vol. 54, No. 3, 1976, pp. 522-523.

[9] N. R. Kressin, J. A. Clark, J. Whittle, M. East, E. D. Peterson, B. H. Chang, et al., "Racial Differences in HealthRelated Beliefs, Attitudes, and Experiences of VA Cardiac Patients: Scale Development and Application," Medical Care, Vol. 40, No. 1, 2002, pp. 72-85.

[10] N. R. Kressin, B. H. Chang, J. Whittle, E. Peterson, J. A. Clark, A. K. Rosen, et al., "Racial Differences in Cardiac Catheterization as a Function of Patients' Beliefs," American Journal of Public Health, Vol. 94, No. 12, 2004, pp. 2091-2097.

[11] L. Cooper-Patrick, J. J. Gallo, J. J. Gonzales, H. T. Vu, N. R. Powe, C. Nelson, et al., "Race, Gender, and Partnership in the Patient-Physician Relationship," Journal of the American Medical Association, Vol. 282, No. 6, 1999 , pp. 583-589. 